\title{
K. POPPER: A FAVOR DE LA VERDAD Y LA RAZON
}

G. RADNITZKY

UNIVERSITÄT TRIER, RFA

0. ¿Cómo podemos lograr una teoría o concepción satisfactoria de la Ciencia?

Como en cualquier otra actividad racional, en la de resolver problemas habrá que comenzar analizando la situación-problema, haciendo un inventario de lo que las filosofías e historias, que nos parezcan importantes, han proporcionado y hacer un examen crítico de ellas. El resultado será el descubrimiento de que están extraordinariamente divididas. Ante tal situación, comenzaré haciendo un bosquejo del sistema en el que me moveré (la filosofía de la ciencia de Popper) para luego proponer una serie de tesis.

\section{Preliminares}

Como cualquier otra actividad humana, la investigación cientifica tiene una dimensión histórica, es cambiante y contingente (véase, por ejemplo, el análisis de la situación-problema del tipo al que nos hemos referido, resultante de la condición históricamente dada al arte). Esa es una observación trivial. Pero por encima de este sentido obvio de historicidad, la Ciencia es un fenómeno histórico en otro sentido más profundo, en un sentido en el que el arte, la literatura, las instituciones políticas, etcétera... no lo son. La Ciencia aspira al progreso cognoscitivo, al aumento y al perfeccionamiento del conocimiento y la idea de progreso cognoscitivo es constitutiva del significado de la ciencia. Es más, es el único campo en el que no puede ponerse en duda que ha habido y hay progreso. Para evitar ser mal interpretado, voy a comentar brevemente este punto. La ciencia tiene una historicidad especial precisamente porque "a diferencia del arte, destruye su pasado" (Kuhn, 1977, p. 345). Puesto que la Ciencia básica tiene como único fin el de mejorar constantemente nuestro entendimiento de la naturaleza, cualquier contribución que aporte más en ese aspecto superará a aquellas otras que hayan conseguido menos. Por tanto, los autores antiguos, incluso los más grandes, no son leídos más que por interés histórico y quizá por el placer de 
ver cómo trabaja una mente genial. En el arte todo esto es diferente. Debido a que el objetivo principal de una obra de arte es conseguir en el receptor una experiencia estética de una clase particular, un estado emocional particular, una figuración de formas posibles de vivir y así sucesivamente, y puesto que hay una variedad enorme de clases de experiencias estéticas, emociones..., las distintas obras maestras aspiran a crear experiencias de estilo distinto. Por tanto, no sería razonable afirmar que Shakespeare superó a Eurípides o R. Wagner a Mozart, etcétera. $\mathrm{Y}$ por eso (y a pesar de todos los logros de las Geistenwissenschaften) hay muchas dudas sobre si entendemos mejor —en el sentido básico de "entender"-, por ejemplo, a Homero que sus contemporáneos.

La relación entre el paso del tiempo y el aumento del conocimiento no es un asunto casual. La Historia de la Ciencia tiene que describir y, en alguna medida, explicar por qué en un caso particular la Ciencia ha progresado asi de hecho. De esa forma, nuestro conocimiento de la Ciencia como fenómeno histórico se hará más profundo. La metodología de la Ciencia tiene que proporcionar instrumentos conceptuales para esa tarea. Otro de sus fines es explicar el concepto de progreso y dar criterios para describir qué desarrollos, de los ocurridos históricamente, son importantes desde el punto de vista del perfeccionamiento de nuestro conocimiento del mundo empírico.

Progreso significa moverse hacia una meta. Un ideal de Ciencia que se muestre generalmente aceptable busca teorias verdaderas que den respuesta a cuestiones cientificamente interesantes. La verdad sola no basta, ya que hay muchas verdades que son triviales. Una cuestión es científicamente interesante cuando responderla correctamente supone una contribución sustancial al perfeccionamiento de nuestra imagen del mundo. Esto vale para la ciencia pura. La ciencia aplicada toma, por definición, sus problemas del exterior de la Ciencia: en la Ciencia aplicada los problemas llegan a considerarse urgentes a consecuencia de decisiones políticas, o sea, debido a la política estatal, o industrial, o... Pero a pesar de eso, los resultados de la ciencia aplicada solo se consideran valiosos para los patrocinadores de la investigación si son suficientemente seguros y probables. Por tanto, el concepto de verdad es todavía de mayor interés, incluso de interés primordial, y de esa forma se convierte en un principio regulativo de toda investigación científica.

\section{Mis tesis}

(1) La ciencia tiene una historicidad evolutiva porque la idea de progreso cognoscitivo es constitutiva del significado mismo de la ciencia, y tiene en el concepto de verdad un estándar objetivo y atemporal. 
(2) La investigación es una actividad racional en cuanto su uso signinifica llevar a cabo, tanto cuanto sea posible, una meta dada: el progreso cognoscitivo. Incluso aunque pueda haber en la investigación aspectos que no sean racionales, la investigación cientifica es, sin embargo, el paradigma de progreso y por tanto, también de la racionalidad deseada en nuestra búsqueda del conocimiento acerca del mundo empirico.

(3) La metodologia de la investigación es un tipo de tecnología ${ }^{1}$ con la que perfeccionar el conocimiento, es decir, un sistema de normas o prescripciones instrumentales diseñadas para aumentar las posibilidades de éxito en la ciencia.

Puesto que la relación entre metodología e historia de la ciencia es uno de los problemas principales de este ensayo, quiero aclarar el concepto de metodologia que utilizo. Como la metodología es considerada como algo afín a la tecnología, la regla metodológica típica podría tener esta forma: "Si te encuentras en una situación del tipo $S$ (la misión del investigador será determinar si se está o no en ese caso) y tu meta es el progreso cognoscitivo, entonces el procedimiento $X$ (por ejemplo, usar ciertos criterios evaluativos para las teorías rivales) es el recomendable, pues si sigues la regla $X$ tus posibilidades de éxito serán mayores que si sigues la regla $Y$ que concurre con ella." Una regla metodológica o prescripción es, por tanto, criticable: su afirmación factual acerca de su utilidad puede ser puesta a prueba. Podrían compararse los esfuerzos del metodólogo con los de un consultor financiero, el cual puede ayudar al cliente a tipificar mejor los tipos de situaciones y puede presentarle sucesos y marcos futuribles, pero que no puede, en definitiva, liberarle de la dificultad y de la misión arriesgada de tomar la decisión.

Por consiguiente, la metodología no es una disciplina empírica descriptiva ni es lógica aplicada. Es normativa, ya que utiliza imperativos hipotéticos en el sentido mencionado más arriba, pero naturalmente no puede dar ningún algoritmo, ya que la investigación nunca es una pura rutina. Por otra parte, tampoco es un proceso caótico que siga la consigna "todo vale". La metodología no limita la libertad del investigador en forma alguna. Al contrario, debería aumentarla al ayudarle a mejorar su autoconocimiento. Dicho sea de paso, en el momento en el que el investigador se mete en una cuestión que no sea puramente rutinaria, actúa como su propio metodólogo, incluso aunque lo haga como un methodologicien malgré lui. Esto ocurre porque constantemente tiene que hacer evaluaciones metodológicas y tomar decisiones, como por ejemplo, la de que esta explicación es mejor que esa, o que esta hipótesis

1 Para la "metodología como tecnología", véanse, por ejemplo, (Radnitzky, 1976), pp. 509-519, especialmente p. 512; (Radnitzky, 1979), p. 250; (Radnitzky, 1980), p. 216; (Radnitzky, 1981) 1.15 "Qué clase de disciplina es la metodología", pp. 65-71. 
ha resultado ser más falible que aquella otra, o que este programa de investigación parece ser más prometedor que su rival y así sucesivamente. Podría ser posible, quizá, que hiciera todo eso con la seguridad con la que anda un sonámbulo. Sin embargo, mientras actúe así, sus reglas de evaluación seguirán siendo implícitas y por eso no podrán ser criticadas y, por tanto, es muy poco posible, o no lo es en absoluto, mejorarlas. Puesto que un investigador no puede evitar tomar decisiones metodológicas, podemos ver una característica negativa de la analogía mencionada entre la metodología y la consulta de inversiones. El inversor puede, si lo desea, delegar en otro las decisiones. El investigador no puede hacerlo; en definitiva, tiene que tomar él mismo y bajo su responsabilidad las decisiones metodológicas necesarias. Un buen ejemplo de esto es la llamada situación de Duhem. En una falsación quedan falsadas las premisas; la cuestión de cuál de ellas es la culpable solo puede decidirla el investigador mismo. Por lo tanto, algunas de las características clave de la metodología son afines a características básicas de las tecnologías, tal y como usamos normalmente este término. Sin embargo, entre la metodologia y lo que considerariamos tipico de una tecnologia hay algunas diferencias. a) En una tecnología típica la meta no sólo viene impuesta desde afuera, sino que puede formularse sin usar la tecnología en cuestión. En el caso de la metodología, la clarificación de la meta, la explicación del concepto de progreso cognoscitivo, así como la crítica de los ideales de ciencia que gobiernan ciertos explicata del "progreso cognoscitivo" sólo puede ser llevada a cabo por la metodología misma. b) Las tecnologías más fiables están basadas en leyes científicamente muy corroboradas. ¿Qué es lo correspondiente a ellas en el caso de la metodología? Sería lo que conocemos o proclamamos conocer sobre el aparato cognoscitivo humano y, por tanto, lo que conocemos o proclamamos conocer sobre la naturaleza general de la realidad empírica. Tal conocimiento lo conseguimos en parte de las ciencias empíricas. Pero una parte esencial la conseguimos por medio de la epistemología y metodología misma. Con lo cual nos enfrentamos al peligro de la circularidad. Pues para decidir qué hipótesis o leyes o teorías están suficientemente corroboradas nos hacen falta reglas de evaluación y esas reglas sólo puede proporcionarlas la metodología. c) Una tecnología basada en el conocimiento científico es un medio muy fiable para alcanzar una meta práctica concreta. Por ejemplo, si fabricamos acero utilizando la técnica propuesta por la tecnología apropiada, entonces podemos contar con que el producto reunirá ciertos estándares específicos. Pero la metodología puede, en el mejor de los casos, facilitar el progreso cognoscitivo, por ejemplo, ayudando a superar algunos obstáculos. Así se han criticado, de hecho, unas cuantas formas de verificacionismo, absoluto y probabilístico, mos- 
trando que el ideal de conocimiento que las gobernaba no podía proporcionar una idea reguladora, debido a que contenía la petición imposible de cumplir de exigir criterios de verdad infalibles. Pero para conseguir el progreso cognoscitivo en un grado importante, con un avance auténtico, necesitamos otros factores: además de método y techné hacen falta unas circunstancias afortunadas (kairos) y muchas cosas más, como sabemos por la historia de los descubrimientos científicos. Sin embargo, la analogía entre metodología y tecnología es fructífera. Además, toda analogía tiene tanto aspectos positivos como negativos.

Tras esta digresión sobre la metodología, puedo ya seguir con el resto de mis tesis.

(4) Toda metodologia se basa en una imagen del hombre, en supuestos metafísicos sobre la capacidad cognoscitiva del hombre, ${ }^{2}$ los cuales no implican una falacia naturalista.

(5) En la filosofia de la ciencia contemporánea hemos conocido dos escuelas de pensamiento totalmente opuestas. Una de ellas ha surgido de la absolutización del principio regulativo de verdad y suele denominarse "Veri-ficacionismo". La otra, el relativismo o historismo, ha surgido de la absolutización de la idea de historicidad de la ciencia, historicidad que comparte con todas las empresas humanas. La existencia de estas dos escuelas de pensamiento ha quedado reflejada dentro de la bibliografía por epígrafes como "la visión heredada" vs. la "nueva filosofía de la ciencia", la escuela "analítico-empirista" vs. la escuela "históricosociológica" 3 de pensamiento y ya, más recientemente, la "tradición abstracta" vs. la "tradición histórica".4

Puesto que toda metodología está incorporada en una teoría epistemológica o en una antropología filosófica, que hace suposiciones sobre la capacidad cognoscitiva humana y sobre el perfil global de la realidad empírica, la oposición mencionada en la metodología se basa en un desarrollo que atraviesa, como un hilo conductor, toda la historia de la filosofía.

(6) Las demandas superambiciosas e imposibles de satisfacer del pensamiento fundacionalista zozobran y caen en un escepticismo radical. $\mathrm{Y}$ subyaciendo a este giro se da un cambio de la imagen del hombre: una visión superoptimista de la capacidad cognoscitiva del hombre acaba convirtiéndose en otra superpesimista.

(7) En la filosofia de la ciencia un ejemplo de esta clase de transformación nos la ofrece la tradición analitica, la escuela positivista-fundacionalista, al dar paso a la visión relativista-historicista. La primera

2 Véase, p.e., (Radnitzky, 1981b) pp. 52, 56 s.

3 Hempel (1979).

4 Feverabend (1981). 
escuela tiene su expresión más cláisca en el Tractatus de Wittgenstein. Este cambio tiene también en Wittgenstein mismo el exponente clásico a nivel personal: en el paso de su filosofía primera [el Tractatus es quizá un experimento mental muy ambicioso: si las proposiciones atómicas (expresión tipo en el lenguaje-PM) proyectan hechos simples, parece factible, entonces, un tipo de lógica trascendental (una simple teoría sea la que sea) que reconstruya la ciencia] a su filosofía tardía de formas de vida monádicas e inconmensurables. Los marcos teóricos no pueden ser discutidos críticamente por la filosofía; si funcionan tienen que ser aceptados. La filosofía primera de Wittgenstein inspiró el positivismo lógico, mientras que su pensamiento posterior ha sido la principal fuente de inspiración para los representantes del relativismo e historicismo como N. R. Hanson, S. Toulmin, T. S. Kuhn y P. Feyerabend, por mencionar solo a algunos de los más prominentes. ${ }^{5}$

Según la postura verificacionista, el ideal de Ciencia considera a la investigación fundamentalmente como la actividad de extender el stock de proposiciones atómicas (preferiblemente en el lenguaje-PM) y de formar proposiciones compuestas a partir de éstas. La justificación se convierte en verificación, es decir, si ignoramos el caso especial del análisis funcional-de-verdad, nos esforzamos por probabilizar, a través de la inducción y sobre la base de un grupo limitado de enunciados básicos consistentemente verdaderos, un enunciado general. En este ambicioso tipo de fundacionalismo - verificacionismo probabilístico _la idea de verificación se apoya en el supuesto de que existe un procedimiento con el que descubrir el valor-de-verdad (o el grado de confirmación) de los enunciados particulares y concretos, y que tal procedimiento es infalible, es decir, que nos garantiza la verdad.

3. Un estudio de la tesis (7) implicaría, entre otras cosas, en primer lugar mostrar por qué tienen que fracasar tanto el programa de verificacionismo puro como incluso - como ya Hume resaltó- su forma más débil de verificacionismo probabilistico, y en segundo lugar, hacer un esbozo general del relativismo.

La primera misión, la de hacer una crítica detallada del inductivismo, fue hecha por Popper en su obra clásica de 1934. Me limitaré aquí a hacer un breve resumen. Aparte ya del llamado problema de la base empírica ("¿hay métodos de averiguar con certeza la verdad de un enunciado observacional?"), al que hubo que contestar negativamente, ese planteamiento no se libra del llamado problema de la inducción: incluso aunque hubiera enunciados básicos cuya verdad pudiera establecerse con certeza, rebrotaría el problema: dado (per impossibile) un conjunto de

5 Este tema está elaborado en Radnitzky (1981c). 
enunciados de observación, de los que podemos estar seguros que son verdaderos, ¿cuándo es racional aceptar un enunciado universal apoyándonos en ellos? Con eso se está ante el problema de determinar el grado de apoyo inductivo o grado de confirmación correspondiente a un enunciado general cuando se da por sentado que los enunciados particulares de observación son verdaderos. El único método que encaja en el programa es inferir el apoyo inductivo de un enunciado general de la verdad de los enunciados deducidos de él. Sin embargo, esta re-transmisión de la verdad, desde la conclusión a las premisas, exige una inferencia ampliadora, que según la lógica estándar, es inválida. Por tanto, surge un problema técnico a la hora de construir una lógica inductiva. Está claro que esa es una tarea matemática; sin embargo, la aplicación de la lógica inductiva origina asimismo un problema filosófico: un cálculo matemático o modelo puede aplicarse a un sistema real solamente cuando el modelo y el dominio tienen suficientes similitudes en aspectos relevantes. La aplicación de la lógica inductiva a un dominio finito es aproblemática, pero carece de interés. Pero si se aplica esa lógica a un dominio que sea, al menos enumerativamente, infinito, la inferencia ampliadora tiene que legitimarse con una meta-regla denominada "principio de inducción". Éste es una asunción metafísica que afirma más o menos que el futuro será como el pasado. Ahora bien, cómo puede justificarse esta asunción? Puesto que es sintética, el procedimiento por el que podría establecerse su verdad tiene que ser o empírico o no-empírico ( $a$ priori). La opción de los métodos empíricos lleva a un regreso infinito o a un círculo vicioso. La opción del apriorismo está excluida por el programa empirista $\mathrm{y}$, además, hay muy pocas esperanzas de justificar un a priori sintético que pueda realizar el cometido del "principio de inducción". ${ }^{6}$

4. La segunda parte de la tesis ` a desmontar era bosquejar el planteamiento relativista. Según esta tesis, el colapso del ambicioso programa del verificacionismo probabilista lleva a la transformación del fundacionalismo en un relativismo, que sostiene no sólo la imposibilidad de justificar la aceptación de una teoría simple sino que sostiene, además, la inviabilidad de la preferencia racional entre teorías. Lo que resta es, entonces, sólo la historia de la ciencia, una historiografía de la ciencia que alega ser metodológicamente neutral y libre, y que intenta encontrar su camino sin ser guiada por la metodología, lo que significa ir sin brú-

6 Popper (1934/1959). Para una visión sintética, véase (Radnitzky, 1979), pp. 217222 y (Radnitzky, 1980), pp. 188-193.

7 Esta influencia se examina con más detalle en (Radnitzky, 1981c), especialmente, pp. 277-280. 
jula -a no ser que use subrepticiamente alguna teoría metodológica$\mathrm{y}$, por consiguiente, no puede producir más que un Musée Imaginaire. La fuente de inspiración de esta clase de relativismo en la filosofía de la ciencia contemporánea es la filosofía tardía de Wittgenstein. Esta ha influido a los representantes más eminentes del relativismo como N. R. Hanson, St. Toulmin y T. Kuhn - que constituye el ejemplo paradigmático- y P. Feyerabend; éstos dos últimos (Kuhn y Feyerabend), trabajando en el estudio de casos históricos, llegaron de forma independiente a posiciones bastante semejantes.

Para justificar la tesis de que el gran auge del relativismo e instrumentalismo en la filosofía de la ciencia contemporánea es una consecuencia de la segunda filosofía de Wittgenstein y también la de que la transformación que Wittgenstein llevó a cabo en el plano personal quedó reflejada en la transformación de tendencias de la filosofía contemporánea de la ciencia, será necesario recordar los caracteres principales de este cambio en la filosofía de Wittgenstein. Consideré, brevemente, su cambio en la concepción del lenguaje, en la de su imagen del hombre, en la de su concepción de la misión de la filosofía y finalmente en su concepto del lenguaje-juego que es central a su planteamiento.

En pocas palabras, el cambio de la concepción de Wittgenstein del lenguaje, desde su primera a su segunda filosofía, transcurre así. La filosofía primera concibe a los esquemas lingüísticos idealizados (formalizados) como pinturas lógicas que pintan, por medio de formas y estructuras de nombres, formas y estructuras de hechos y objetos simples. La filosofía final concibe al lenguaje como actividades humanas integradas en otras actividades humanas por medio de incontables clases diferentes de usos de las palabras. Ese cambio puede ilustrarse con la transformación experimentada en conceptos clave desde el Tractatus (en adelante, ' $T$ ') a las Investigaciones Filosóficas (en adelante, 'PI'): al atomismo, elementarismo, le corresponde ahora el lenguaje-juego (PI .7.65); a las proposiciones atómicas ( $T$ 4.2) corresponden "nuestros juegos-de-lenguaje simples y claros" (PI 130); a las figuras o diseños (T 2,1-2,2) corresponden instrumentos (PI 569), conceptos (PI 569), descripciones (PI 291); a los nombres y objetos que son nombrados ( $\mathrm{T} 3,22)$ corresponden usos (PI 30-43); a los hechos, a lo que "se comporta así y asi" (T 4,5) corresponde "así lo hacemos", es decir, acción y significado son previos a los hechos.

La transformación de Wittgenstein acerca de la tarea de la filosofía -que ha influido profundamente en la visión que tienen de la tarea de la Filosofía de la Ciencia los relativistas contemporáneos- parece estar intimamente ligada a su transformación de la imagen del hombre. Ciertamente, un cambio en la imagen del hombre parece subyacer al cambio 
de su concepción de la filosofía en la última etapa. En la auto-concepción del hombre, el centro de gravedad ya no se pone en el hombre en cuanto conocedor, sino en el hombre moviéndose en un mundo de relaciones-de-significado. La situación primaria del hombre no es ya el conocer sino lo que nos resultan fenómenos con significado. Hay situaciones vitales y las hay significativas para nosotros como partes de nuestra vida. Tienen el status de un a priori práctico, en el que proporcionan el contexto de presuposiciones dentro del que la Ciencia, como todas nuestras otras actividades, existe. Y la ciencia no tiene un status privilegiado. Esto es algo que Feyerabend subraya especialmente, como consecuencia de la filosofía de Wittgenstein.

En su filosofía primera veía la misión de la filosofía como un tipo de "reconstrucción" hecha por medio de un mapa de hechos y de hechos posibles, construida por signo-diseño con la ayuda de un esquema lingüístico gobernado por la gramática lógica (en lugar de la gramática ordinaria) ( $T$ 3.201). La filosofía posterior considera que la tarea consiste en efectuar "readaptaciones" (PI 92) que nos posibiliten "disponer de una visión clara del uso de nuestras palabras" (PI 122). Filosofar es describir. Y no hay una sola descripción correcta sino descripciones dispuestas para eliminar ciertas confusiones ocasionadas por el mal uso del lenguaje: tenemos que contentarnos con formas de ver que eliminen el encantomiento de nuestra.inteligencia por nuestros lenguajes imperfectos. ¿Cuál es la concepción de la realidad en esta filosofía final? La realidad es colocada en la experiencia inexpresable del momento presente. (Como en el budismo zen, lo que tenemos que comprender es que no hay nada entre nosotros y el mundo.) La filosofía se convierte en una mayeútica del silencio. "El descubrimiento auténtico es el que me hace capaz de dejar de hacer filosofía cuando quiero. El que lleva la filosofía a la paz, de forma que no esté atormentada por cuestiones que la ponen a ella misma en cuestión" (PI 133).

El concepto del lenguaje-juego de la filosofía tardía de Wittgenstein influyó decisivamente en la concepción sobre las tradiciones o estilos en la ciencia y también la concepción de la ciencia misma adoptada por los escépticos modernos. En los estudios sobre la filosofía tardía de Wittgenstein, el concepto de lenguaje-juego se presenta a menudo como si su rasgo más importante fuera el de proporcionar una forma de ver el mundo. Pero para Wittgenstein un lenguaje-juego es, primera y principalmente, una manera de actuar, “... no es un tipo de ver por nuestra parte; es nuestra acción lo que yace en el fondo del lenguaje-juego" ( $O n$ Certainty, p. 204). "Lo que tiene que ser aceptado, lo dado", las formas de vida, son fundamentalmente patrones de actividades con significado. Una forma de vida es algo que nosotros hacemos, que no tiene otra jus- 
tificación más allá de sí misma. No tiene justificación y no la necesita porque las formas de vida definen lo que tiene sentido hacer y no hay otras maneras de dar sentido en cuyos términos puedan ser cuestionadas o justificadas. De acuerdo con este planteamiento, ya no se ve a la ciencia como una representación del mundo que nos ayuda a entender la naturaleza sino, primariamente, como una actividad humana, que es una más entre una serie de actividades $\mathrm{y}$, paradójicamente, se ve a la "Ciencia" como una actividad que no se ocupa de la realidad. Sin embargo algunos seguidores de Wittgenstein consideran que la "Ciencia" es "una actividad humana muy importante", como por ejemplo Henri LeRoy Finch. Otros, como P. Feyerabend, dudan si la "Ciencia" disfruta de una posición privilegiada entre otras actividades humanas de interés. Después de esta brẹve visión general de la transformación de la filosofía de Wittgenstein, que se convirtió en la fuente de inspiración del relativismo/instrumentalismo en la filosofía de la Ciencia contemporánea, podemos continuar ya nuestro examen del planteamiento relativista.

Tesis 8: La característica más importante de la escuela relativista me parece que es que incorpora en la metodologia de la historiografia misma el marco conceptual de una escuela alemana concreta: el historismo según fue introducido por L. von Ranke. El relativismo histórico o historismo sostiene que, puesto que individuum est ineffabile, cualquier intento por explicar en una narración un suceso por medio de una ley general de varias disciplinas, no puede hacer justicia a su carácter único, y por eso "todos los sucesos son equidistantes de Dios", todos los marcos son igualmente buenos, y no hay ninguna evaluación racional posible. Sencillamente no hay estándares de evaluación que sirvan para establecer puentes entre las tradiciones. Por consiguiente, para ser "cientifico" (en el sentido del ideal positivista) el historiador tiene que restringirse a hacer una descripción de la serie de épocas, de estados, de culturas, ecétera...8 $\mathrm{El}$ modelo de sistemas que se autocontienen, sucediéndose entre sí más casualmente que históricamente, inconmensurables con cualquier otro sistema e incomprensibles cuando son considerados desde otro sistema, fue usado por Voltaire y Spengler en historia, por Malinowsky en antropología y encontró finalmente una puerta de entrada en la metodología de la ciencia a través de la filosofía tardía de Wittgenstein.

El historismo es básicamente un relativismo ahistórico y no evolucionista. Florece sobre lo que Popper ha llamado "el mito del sistema (o

8 Los escritos sobre el historismo son abundantes, particularmente en alemán e italiano. Para una visión breve, véase, por ejemplo, Munz (1977), pp. 62 ss. El tema se trata con más detalle en el artículo de Munz "Evolution and Equidistance" (próximo a publicarse). 
marco)": la creencia de que somos prisioneros de los marcos conceptuales y que, en el mejor de los casos, sólo podemos dar un salto irracional - parecido a una conversión- desde un marco conceptual a otro. Si llevamos al historismo hasta su última consecuencia, éste tiene que negar el progreso también en la Historia (res gestae) de la Ciencia. Esto muestra con particular claridad que aquel rechaza la idea de la evolución o aumento del conocimiento. Al afirmar que todos los sucesos (L. von Ranke), o todos los paradigmas ( $T$. Kuhn), o todas las tradiciones ( $P$. Feyerabend) son, para usar la famosa frase de Ranke, "equidistantes de Dios", está introduciendo subrepticiamente un punto de vista evaluativo que absolutiza el pluralismo. De esta forma se pierde el problema de evaluar desarrollos científicos respecto a aspectos concretos. La idea reguladora de verdad queda perdida junto con el problema de la evaluación y con eso se abandona el realismo. Sin la asunción de un realismo ontológico y epistemológico es imposible ofrecer un explicatum adecuado de la idea de progreso científico, y sin criterios de progreso (incluso aunque su uso se base en indicaciones falibles) la historia de la ciencia (historia rerum gestarum) no puede conseguir más que el Musée Imaginaire -que el historiador piensa que es la única cosa que puede hacer "cientificamente", es decir, sin poner en peligro el ideal positivista de la ciencia.

En la historiografía misma el historismo ha sido capaz de conseguir casi un monopolio académico y en los últimos tiempos se ha disfrazado con vestimentas nuevas sacadas del departamento de rebajas del emperador, tales como el estructuralismo, la cliometría, etcétera. ${ }^{9}$ En la metodología de la Ciencia contemporánea nos encontramos con un desarrollo parecido: el historismo se importa desde el estructuralismo. El ejemplo más prominente de esto sería el desarrollo desde el historismo, tal y como lo practica $T$. Kuhn, al instrumentalismo (estructuralismo) de W. Stegmüller. La reconstrucción de Sneed-Stegmüller de la teoría del cambio de Kuhn, el planteamiento "no-enunciativo" o "estructuralista" de las teorías, es una combinación interesante, y admirable en su claridad y precisión, del planteamiento formalista del positivismo lógico y del historismo. Según la teoría de Sneed-Stegmüller adscribir un valor de verdad a una teoría científica no tiene sentido. Puesto que una teoría no es más que un formalismo con unas pretensiones de aplicación, la evaluación de la teoría depende de si estas aplicaciones han tenido éxito o no, porque es la "aplicación pretendida" la que proporciona la única conexión entre la teoría y la experiencia. Lo mismo que Kuhn y Lakatos, quienes afirmaban que la historia de la ciencia demuestra que durante la "Ciencia normal" un paradigma (Kuhn), o el núcleo duro de un pro-

9 Peter Munz en "Evolution and Equidistance". 
grama de investigación (Lakatos), no pueden ser falsados, el planteamiento no-enunciativo minimiza también el papel de la falsación. Sin embargo, quitar a la falsación su capacidad de morder equivale a rehusar aprender de la experiencia y, por consiguiente, la estrategia implicada en este planteamiento no puede facilitar el aumento del conocimiento. ${ }^{10}$

El rasgo decisivo común entre el planteamiento "no-enunciativo" o "estructuralista" de las teorías (llamado por Feyerabend la "sneedificación de la ciencia"), la filosofía básicamente inductivista de la ciencia de Kuhn y el "anarquismo epistemológico" de Feyerabend es el instrumentalismo. Este absolutiza una de las funciones de las teorías científicas. Naturalmente las teorías son, entre otras cosas, instrumentos que sirven para hacer predicciones y explicaciones. Pero ¿cómo pueden conseguir éxito explicativo y predictivo si no tienen, al menos en alguna medida, éxito en la descripción o representación de algunos aspectos de la realidad con suficiente certeza? Al abandonar el postulado del realismo, el instrumentalismo ha negado también la verdad como principio regulador de la investigación científica. Esa negación lleva a un planteamiento historista de la historiografía de la ciencia y, en definitiva, lleva - como en el caso de P. Feyerabend- a negar la posibilidad de cualquier metodología de investigación. De esta forma la idea de verdad ha sido totalmente suplantadas por la idea de la historicidad, pero historicidad en el sentido no-evolucionista y, en último caso, ahistórico del historismo (P. Munz).

5. Puesto que la visión de Kuhn ha tenido una influencia tan enorme, vale la pena examinarla más detenidamente. ¿Qué planteamiento metodológico subyace en la historiografia de la ciencia de Kuhn? El planteamiento de Kuhn se caracteriza por el fundacionalismo, el positivismo y el historismo. Fundacionalismo: se concede al conocimiento proporcionado por la historiografía de la ciencia kuhniana una posición epistemológicamente privilegiada: es el único conocimiento racional que no es una convención (lo que equivale al dogmatismo) y al cual se puede apelar para mostrar que todas las demás formas de conocimiento racional son convenciones (lo cual equivale al escepticismo, por lo que podría calificarse de "escepticismo dogmático"). Positivismo: en su historiografía de la ciencia — que, según se afirma, está exenta de metodologiaKuhn observa y describe "hechos puros" de la historia de la ciencia (res gestae). (Aunque, según la conclusión que Kuhn saca de su análisis, su propia historiografía debería estar tan dirigida por el paradigma como todas las otras.) A partir de su historiografía de la ciencia, basada sobre 
el cimiento de los hechos históricos, se siente con derecho a derivar inductivamente (¿cómo si no?) un tipo de metodología general de la ciencia que es, además, simultáneamente descriptiva y prescriptiva. ${ }^{11}$

Historismo: la sucesión de cambios de paradigma representa un proceso "sin la ventaja de tener una meta",12 es decir, sin la ventaja de la idea de verdad o, en la práctica, del concepto comparativo de parecido a la verdad (zutreffendere Darstellung) como un principio regulativo. Por consiguiente, Kuhn no puede usar el progreso científico como el rasgo distintivo que hace a la ciencia un fenómeno histórico, es decir, que le da historicidad en un sentido superior y por encima del sentido trivial en el que cualquier actividad tiene historicidad. De acuerdo a la exposición de la historia de la ciencia presentada por Kunh, las teorías son convenciones que se suceden unas a otras, con lo que la única guía en la elección de la teoría paradigmática es que se espera que la nueva convención sea capaz de enfrentarse a las dificultades surgidas en la vieja y que no pudieron resolverse dentro de ella. Esto es lo que Popper ha llamado "el mito del marco o sistema": la única manera de salir de tal sistema (cerrado) es un salto irracional, afín a una conversión, para adoptar otro sistema (paradigma). A la cuestión de "cuándo es racional abandonar un paradigma para pasar a otro", la única respuesta de Kuhn es que, a veces, se da un consenso en la comunidad cientifica. ${ }^{13} \mathrm{Kuhn}$ describe la resolución de las revoluciones científicas, por medio de la adopción de un nuevo paradigma, como la "selección mediante el conflicto, dentro de la comunidad científica, de la manera más apropiada de practicar la ciencia en el futuro". ${ }^{14}$ Sin embargo, como señala Hempel, no está claro cómo vamos a construir "la afirmación de que la elección teórica, según es llevada a cabo por la comunidad cientifica, selecciona la manera mds apropiada de seguir practicando la ciencia, especialmente en ausencia de objetivos definidos que pudieran proporcionar algún criterio explícito de evaluación". ${ }^{15}$ Yo diría que, una vez más, el uso de la metáfora de la evolución queda aquí como una reverencia hueca al pensamiento evolucionista. A pesar de todo, Kuhn, y especial-

11 (Kuhn, 1970), p. 237.

12 (Kuhn, 1962), p. 172. La doctrina historista sostiene que las cadenas de sucesos forman en la narración dibujos de desarrollo "orgánico" (P. Munz). Ellos apelan asimismo -véase Kuhn_ a una metáfora extraída del pensamiento evolucionista a pesar del hecho de que su planteamiento general no es evolucionista: Kuhn ha rechazado el concepto de progreso del que se pueden derivar los estándares objetivos de evaluación y Feyerabend señala explícitamente que él usa la palabra "progreso" en el sentido en el que la usan los diferentes grupos cuyas actividades de investigación estudia; Cf., (Feyerabend 1972) p. 168.

13 (Radnitzky-Anderson 1978), p. 8.

14 (Kuhn 1962), p. 171.

15 (Hempel 1979), p. 300. 
mente sus seguidores, creen que pueden concluir de su historiografía de la ciencia, debido a que está basada en "hechos" históricos, un planteamiento en la metodología general de la ciencia que muestre, cuando menos, que el falsacionismo no es capaz de servir de guía a la historiografía de la ciencia, ni de dar un conjunto practicable de prescripciones instrumentales al investigador.

Como la sociología del conocimiento, la posición de Kuhn es fácilmente reducible al absurdo. ${ }^{16}$ Según su propia teoría, la historiografía de la ciencia, que naturalmente incluye la propia versión kuhniana de ella, no es en último caso más que una convención incapaz de sostener cualquier metodología general de la ciencia, excepto naturalmente la postura metodológica que subyaga a la construcción.

6. ¿Qué decir, entonces, de la historiografia de la Ciencia? En primer lugar, una aclaración general sobre la metodología de la historiografía. Debe evitarse tanto el historismo como el historicismo.

Tesis 9: En la metodología de las ciencias históricas podemos detectar un fenómeno parecido al ya mencionado anteriormente de la transformación del fundacionalismo en escepticismo, pero que va en sentido opuesto: el historismo se vuelve historicismo, es decir, el relativismo se convierte en una nueva clase de dogmatismo. Esta transformación comienza con la tesis historista de que las series de sucesos presentados en las narraciones históricas ofrecen un desarrollo "orgánico". Esta tesis se transforma en la doctrina historicista que declara que los sucesos históricos son estructurados por una ley única de la historia. El historicismo es una versión secularizada del pensamiento escatológico que convierte a los historicistas en confidentes de la providencia. (Una visión historicista de la historia de la ciencia es, por ejemplo, la de que el progreso cientifico tiene que ser lineal y acumulativo, o la de que la ciencia alcanzará un estado final o se acercará a tal estado). El proceder posterior del historicista es, entonces, un largo círculo. En la selección de sus datos se guía por su ley histórica y luego usa esos datos para demostrar la verdad de su ley (P. Munz). Como la historia de cualquier otra actividad, la historia de la Ciencia no puede transcribirse simplemente de la realidad. Naturalmente, L. V. Ranke, el padre del historismo, tenía razón al declarar que los historiadores tratan de averiguar lo que sucedió de hecho. Pero la historia no consiste en relatar cómo una serie de sucesos se suceden temporalmente. Tienen que ponerse y armarse conjuntamente.

Tesis 10: Para empezar su investigación, el historiador de la Ciencia

16 La crítica de Popper a la sociología del conocimiento en Popper (1945), vol. II, pp. 208-216, 223 s., 242,251 s., 267. 
necesita un mecanismo de selección con el que averiguar qué sucesos tienen significado, son "cientificamente" importantes, es decir, importantes de cara al aumento del conocimiento. Y solamente la metodologia puede dar al historiador de la ciencia esos criterios de evaluación. (La metodología de la física puede orientar a la historiografía de la física $\mathrm{y}$ así sucesivamente.) Tiene que describir los desarrollos importantes y explicar por qué un suceso concreto, un suceso que es importante para el aumento del conocimiento, ocurrió como ocurrió y no de cualquiera de las otras formas posibles. Para unir un evento a otro tiene que usar leyes generales de varias disciplinas. Las necesita para conectar una condición inicial y su efecto - tanto en la historia de la ciencia como en la historia de cualquier otra actividad. El establecimiento de esos lazos depende de las leyes generales que el historiador considere probables, plausibles. ${ }^{17} \mathrm{La}$ historia de la ciencia no es una excepción. En suma, que el historiador de la ciencia necesita, además de la metodología de la historiografía, la metodología de la disciplina en cuestión cuya historia está reconstruyendo. $Y$ lo más decisivo de todo, necesita criterios de evaluación. Tanto la metodología de la disciplina en cuestión como la metodología de la historiografía están ellas mismas enclavadas en una metodología general de la investigación. Esta metodología determina, entre otras cosas, la imagen que el historiador de la ciencia tiene de su campo, de la disciplina que es el objeto de sus investigaciones, y de la ciencia en general. Por eso, toda investigación histórica de la ciencia está influida por los presupuestos metodológicos que el historiador —explícita o implícitamente- hace. ${ }^{13}$

¿Cómo puede un metodólogo usar la historia de la Ciencia? Salvo que él mismo se convierta en un historiador, tiene que basarse en las exposiciones presentadas por los historiadores de la ciencia. Cada una de esas exposiciones del desarrollo factual de la ciencia está impregnada por un planteamiento metodológico, es decir, por el punto de vista metodológico adoptado por el historiador o aplicado, subrepticiamente, por él. Por consiguiente, el metodólogo debe averiguar, en primer lugar, qué clase de gafas ha usado el historiador al hacer el estudio del caso que al metodólogo le parece interesante o digno de atención. Después tiene que dejar a un lado la influencia de esas gafas. Eso sólo podrá hacerlo analizando el texto y extrayendo de esta forma las lecciones que el estudio del caso pueda darle acerca del ejemplo de progreso cognoscitivo considerado. ${ }^{19}$

17 Véase (Munz 1977), (más arriba).

18 Esta dependencia se examina y ejempliza en Antiseri (1981), especialmente pp. 354-860. Véase también (Barone, 1980).

20 Para una visión general de la metodologia popperiana, véase (Radnitzky 1976) y 
7. La alternativa $o$ trabajar con el planteamiento fundamentalista $o$ el escepticismo (relativismo sociologista-historicista o instrumentalismo), debe parecer un dilema a aquellos que están metidos en el estilo de pensamiento fundacionalista, es decir, a aquellos que se adhieren a un concepto de conocimiento, según el cual solamente un determinado conocimiento, el de los enunciados cuya verdad ha sido establecida por métodos infalibles de determinación, es conocimiento auténtico. Cada rama de este dilema alegado se debe a una imagen falsa del hombre. En el caso del estilo fundacionalista hay una visión superoptimista de la capacidad de nuestro conocimiento y en el caso del estilo escepticista hay una visión superpesimista. Si se reemplaza cualquiera de esas dos imágenes falsas del hombre por una autoconcepción realista de este como especie, el hechizo se rompe: aunque el conocimiento humano de la realidad empirica es, por principio, falible, el progreso cognoscitivo, en el sentido de acercamiento a la verdad, es sin embargo posible y tenemos algunos ejemplos sorprendentes de tal progreso en la historia de la ciencia. Aunque han existido algunos predecesores en la historia intelectual, desde Anaximandro (a quien debería canonizarse como patrono del racionalismo crítico) pasando por C. S. Peirce, quien introdujo el 'término "falibilismo". en el siglo xIx, hasta Lukasiewicz y algunos otros, el avance decisivo del falibilismo se dio gracias a la filosofía de la ciencia de Popper. ${ }^{20}$ El falibilismo se ocupa de los métodos de determinación del valor de verdad de enunciados concretos o, más exactamente, del procedimiento que puede ser usado para averiguar si hay buenas razones a favor de nuestra conjetura de que una teoría se acerca más a la verdad que otra, es decir, es más acertada en la descripción o representación de determinados aspectos de la realidad. Se reconoce la historicidad fundamental, la relatividad temporal de todos los informes de la contrastación empirica. El falibilismo no solo es compatible con, sino que presupone la idea de verdad (atemporal) al implicar la idea de error. Sin el concepto de verdad la idea de "contrastación empírica" quedaría también sin significado. ${ }^{21}$ (Incluso la idea de basar la evaluación teórica sobre las "aplicaciones con éxito" implicará eventualmente la idea de verdad, ya que implicará predicciones y una predicción con éxito es una predicción correcta.)

La elección de teorías es, en la metodología popperiana, un proceso racional, o puede y debe serlo. La conjetura de que $T_{2}$ es superior a $T_{1}$

19 (Andersson 1981) da un ejemplo.

(Radnitzky 1981a). Para una elaboración de la tesis de que esa alternativa mencionada tiene que presentarse como un dilema a los fundacionalistas, véase, por ejemplo, (Radnitzky, 1979) y (Radnitzky 1980).

21 Véase, por ejemplo, (Radnitzky 1980), p. 202. 
en determinados aspectos específicos, principalmente en su parecido comparativo a la verdad (estándar atemporal), se basa en buenas razones. La situación en la elección entre teorias puede resumirse así: la base es la evaluación metodológica de los logros anteriores de las dos candidatas evaluadas en cuanto a su éxito y su fracaso en la explicación y predicción. En mi opinión, estos informes de los logros anteriores de las dos rivales deberían ofrecerse en una forma particularizada y detallada $y$, además, debería darse a cáda aspecto o ítem un índice de la "importancia científica" de la cuestión a la que intenta responder, es decir, una estimación de la contribución que una respuesta correcta a esa cuestión haría al avance de la disciplina de que se trate. ${ }^{22}$ El concepto de grado comparativo de corroboración intenta resumir precisamente ese aspecto del logro previo (pero no puede dar explicación de la "importancia científica" de las cuestiones que se han intentado responder). Se usa como un indicador falible pero objetivo del logro previo. Con la ayuda de ese indicador podemos formular buenas razones a favor o en contra de que una cierta teoría concreta $T_{2}$ es superior a sus rivales en su capacidad para describir más correcta y acertadamente ciertos aspectos de la realidad. No se exigen más que buenas razones. El mismo veredicto se mantiene como una hipótesis conjetural. Popper nunca intentó demostrar que una teoría es verdadera o probable en un determinado grado y no se da una inferencia lógica desde los informes acerca de en qué medida o en qué grado las teorías rivales han aguantado la critica rigurosa, a la conclusión de que una teoria $T_{2}$ es superior a $T_{1}$. Por otra parte, la relación entre el enunciado que da a una teoría un cierto grado de confirmación o de apoyo inductivo o probabilidad, sobre la base de determinados datos, y el conjunto de enunciados que formulan los datos es de una implicación lógica del tipo de una inferencia amplificadora desde la conclusión a las premisas. Por consiguiente, hay una gran diferencia entre el concepto de grado comparativo de corroboración de Popper y el concepto inductivista de grado de confirmación, y por tanto, hay también una gran diferencia en la función asignada a cada uno de ellos en la evaluación de teorías.

En la metodología de Popper nunca se hace el intento de probar que una teoría es verdadera o de darle una probabilidad. De acuerdo a su metodología, no existe un procedimiento objetivo para determinar que una teoría concreta tiene un grado específico de probabilidad o de apoyo inductivo. Naturalmente, nosotros poseemos una confianza subjetiva en que las regularidades descritas por una teoría altamente corroborada serán válidas también en el futuro. Pero esta creencia subje-

22 Este tema se elabora algo más en (Radnitzky 1979) y (Radnitzky 1980). 
tiva, esta convicción, no garantiza ningún significado metodológico. Según la metodología de Popper, esta convicción subjetiva no se considera racional porque se sostenga que la teoría tiene un alto grado de apoyo inductivo; se considera racional adoptar esa convicción y actuar sobre ella porque la teoría ha sido contrastada severamente. No se ha intentado establecer la probabilidad de la teoría por medio de la búsqueda de casos positivos; más bien, buscamos posibles casos negativos y defendemos que esta teoría ha superado mejor la contrastación empírica que su competidora y, por consiguiente, suponemos que se asemeja más a la verdad que sus rivales.

Esa conjetura seguirá siendo, por principio, revisable, cuando y solamente cuando haya razones suficientes que justifiquen esa reconsideración. Por esta razón, y solo por esa razón, es racional, por lo que concierne a las aplicaciones tecnológicas, moverse con esa teoría y usarla para resolver problemas prácticos. Esa acción y la convicción que subyace en ella es racional, incluso la única línea de acción racional - pero la convicción es, sin embargo, metodológicamente irrelevante.

Se ha recalcado que las buenas razones mencionadas anteriormente deben ser ellas mismas tan falibles como todo lo demás, de forma que la falsación sea por si misma y por principio falible. Los fundacionalistas afirman a menudo que, si no hay determinados enunciados básicos, entonces la idea de falsación no tiene sentido. Asi lo hace, por ejemplo, Thomas Kuhn. ${ }^{23}$ (Quizá debería usarse un término técnico como "discorroboración" para contrarrestar el inductivismo oculto del uso ordinario, a causa de que la tendencia fundacionalista queda reforzada por el uso, en el lenguaje ordinario, de "falsación" y de "conocimiento".) Es llamativo que aquellos que reconocen la interpretación fundacionalista es insostenible no hayan sido capaces, sin embargo, de liberarse ellos mismos de la corriente subterránea fundacionalista mencionada anteriormente, e incurran en el vicio opuesto al interpretar la falsación y "los enunciados básicos" como convencionales. Éstos han caído en la trampa escéptica. ${ }^{24}$

Por consiguiente, a pesar de la falibilidad fundamental de los métodos de determinación del valor de verdad de unos enunciados descriptivos concretos (o de una parte de cualquier posición, visión del mundo, 'hipótesis, etc.), la verdad sigue siendo uno de los principales principios reguladores de la investigación científica. Sin embargo, la situación metodo-

23 Esta es la visión adoptada por Kuhn; cf. (Kuhn 1970), p. 15: "¿Qué es la falsación si no es una disprobación concluyente?"

24 Para la relación entre fabilismo y falsacionismo, con una atención especial a la obra de T. Kuhn, véase (Anderson, 1981). 
lógicamente más interesante en la evalución de teorías es aquella en la que debe establecerse una preferencia racional entre dos teorias rivales que tienen "dificultades", es decir, que tienen que ser, expresándolo rigurosamente, consideradas falsas, o expresándolo más cautamente, que se ha conjeturado que son falsas porque de momento han sido discorroboradas. Por consiguiente, en la práctica el concepto de parecido a la verdad, o más exactamente, el concepto comparativo de "ser más parecida a la verdad que la teoria o teorías rivales" sirve como un principio regulador. Las dificultades para introducir el explicatum "verosimilitud" por medio de una definición formal son de sobra conocidas. Depende del uso del concepto de clases de consecuencias y, en mi opinión, se producen principalmente por culpa del instrumento empleado en esa definición, los sistemas lingüísticos idealizados o formalizados. A pesar de todo eso, la idea intuitiva de que la teoría es una descripción más o menos acertada que otra teoría rival es totalmente indispensable tanto en la metodología como en la vida diaria.

El parecido a la verdad, comparativamente más grande que el de una rival, es uno de los signos principales de progreso cognoscitivo. Existe otro, derivado de un segundo aspecto del fin de la investigación científica. La ciencia no sólo aspira a crear teorías verdaderas de la realidad empírica sino también busca teorías que den respuesta a cuestiones "científicamente interesantes". Y para Popper el progreso consiste, esencialmente, en ir de problemas profundos a otros todavía "más profundos". Por consiguiente, mi propuesta es que deberíamos distinguir claramente tres cuestiones distintas: 1) lo que la teoría afirma y cuánto afirma, es decir, su contenido empírico; 2) si lo que esa teoría afirma es verdadero o más parecido a la verdad que lo afirma la mejor hipótesis rival acerca de esos aspectos concretos de la realidad, y 3) preguntarnos si lo que se afirma, en caso de que sea correcto, nos capacitaría para mejorar sustancialmente nuestra imagen del mundo o del hombre. Esta última consideración nos lleva fuera de la ciencia, puesto que el perfeccionamiento continuo de nuestra imagen del mundo es un fin extracientífico - un fin que puede, sin embargo, darnos un segundo principio regulador de la actividad teórica.

8. En contraposición a la "visión heredada", es decir, al planteamiento formalista del empirismo lógico, la alternativa popperiana recalca en todo momento la historicidad, historicidad en el sentido de crecimiento evolutivo. Lo hace asi en su modelo básicamente temporal del falsacionismo: pasos creativos e innovativos para encontrar falsadores potenciales, alternados con pasos en los que se examinan esos falsadores poten- 
ciales para averiguar si pueden ser tratados o convertidos en verdaderas hipótesis falsadoras. ${ }^{25}$

La epistemologia evolucionista puede considerarse como una extensión del falsacionismo. ${ }^{26}$ El aumento del conocimiento es considerado como una continuación de la selección natural. En el nivel de los órganos biológicos del conocimiento, órganos de percepción, aprendizaje, cognición y lenguaje, los órganos son considerados como solucionadores de problemas. ${ }^{27}$ Por ejemplo, aquellos animales a los que sus ojos o sus cerebros les llevasen a errar normalmente desaparecerían al tener el percibir, el conocer aspectos relevantes del entorno con suficiente grado de validez, un valor continuo de supervivencia para los animales. La no-casualidad, la selección retentiva de las variables biológicas produce categorías o facultades de percepción, cognición, etc., que se consideran a priori para el individuo, pero a posteriori para la especie y que no llevan adscrita una validez a priori sino que son vistas como solucionadores aproximativos de problemas adaptados a un entorno previo, pero no necesariamente a los entornos futuros. Este tipo de selección se concibe en continuidad con la preferencia racional de teorías en la investigación científica.

La perspectiva de sistemas téóricos de la epistemología evolutiva sigue un patrón básico: en el proceso de evolución se da un incremento del orden y una acomodación de un sistema a otro. Este crecimiento, este proceso evolutivo o "aumentativo" es producido por un proceso repetido de variaciones fortuitas (impredictibles) y no metódicas, "ciego" pero no casual (puesto que en el plano biológico están gobernadas por leyes de la biología molecular y en el nivel verbal por las leyes de la lógica necesaria), de momentos innovativos que se influyen incesante y repetidamente con lo no-casual, con la selección retentativa y duplicación. Puede mostrarse ahora que el patrón funciona y ha estado funcionando en los distintos planos y niveles desde el origen de la vida. Puede ilustrarse por la historia desordenada e impredecible de mutaciones casuales en el nivel de la biología hasta las invenciones de la inteligencia humana, por medio de la selección retentiva incorporada en los órganos en cuanto solucionadores de problemas, hasta la historia de la ciencia. La selección natural ${ }^{28}$ es tanto un ejemplo como una fuente de inspiración para este modelo de sistemas teóricos. El modelo ayuda a explicar un amplio campo de fenómenos desde el aprendizaje por ensayo-error hasta proce-

25 Cf., p. e., (Radnitzky, 1976) y (Radnitzky, 1981a), especialmente pp. 75 y 81 (figuras 1 y 2).

26 El lector debe remitirse concretamente a (Popper, 1972), cap. 7, y Campbell (1960) (1974a) (1974b) (1977) y al análisis agudo de (Bartley, 1976).

27 (Pepper, 1972), p. 70.

28 Incidentalmente, Darwin usó primero la expresión "lucha de la naturaleza" (del "Toda naturaleza es lucha" de Hobbes). Después, y por la influencia del libro de 
sos iterativos en la computación, formación de cristales, el sistema de inmunización selectiva y el comportamiento animal, según es estudiado por la etología comparativa y los procesos de investigación mismos. Popper señala que, en el nivel biológico, el mecanismo de selección opera sobre los individuos. En este nivel son los individuos quienes son eliminados por medio de la "eliminación de errores", mientras que en el nivel del conocimiento humano lo que es eliminado son las teorías conjeturales.

El núcleo de la metodología de Popper es el modelo de la solución racional y acertada de problemas: conjeturas que se combinan con la crítica por medio de la contrastación empírica rigurosa. Este modelo se ocupaba, en un principio, del proceder de la investigación científica. Más tarde se descubrió que el modelo era también aplicable fuera del contexto humano. Por ejemplo, podía usarse para explicar el comportamiento animal; y enriquecido con conceptos de la economía podía aplicarse también a la forma en la que un individuo o una población maximaliza su éxito en términos de propagación. ${ }^{29}$ De todo esto puede concluirse que el modelo posee también un gran poder heurístico fuera de la filosofía de la ciencia y de la teoría del conocimiento. Esto parece dar un cierto tipo de apoyo al modelo. El tipo de relación existente entre el estudio biológico comparativo del aparato cognoscitivo, de una parte: y la epistemologia y filosofía, de la otra, es de interacción. Cuando en el contexto de la "epistemología evolucionista" el éxito es descrito en términos de supervivencia, propagación..., eso es biología. Pero cuando tal éxito se aplica con referencia a que las percepciones de cuestiones se han hecho más verídicas o las "hipótesis" (esquemas de comportamiento) más parecidas a la verdad, entonces, naturalmente, se ha recurrido a la evaluación epistemológica. Si la epistemología evolucionista propone que el "a priori sintético" sea a posteriori para las especies y no eterno, eso es una crítica implícita a ciertas epistemologías filosóficas. Sin embargo, y dado que no se afirma que la evaluación epistemológica sea derivable de la investigación biológica, no tiene sentido la acusación,

Malthus, la cambió por "lucha por la existencia". Posteriormente introdujo la expresión, más adecuada, "selección natural"; pero en la sexta edición de los Origenes la sustituyó por "supervivencia del más adaptado" (de Spencer), expresión que parece ser una fórmula vacía ya que el que sobrevive lo hace porque está adaptado y que está adaptado lo sabemos por el hecho de que ha sobrevivido. Dicho sea de paso, en. las 70000 palabras del texto de Darwin, el término "evolución" aparece sólo once veces. Por otra parte, Spencer lo usó a menudo y lo popularizó. Sin embargo, no nos ocupamos aquí del problema de explicar la "selección natural" o la "evolución" en la teoría biológica $y$, desde luego, no pretendemos hacer una exégesis del texto de Darwin, antes bien, tales conceptos nos sirven como instrumentos heurísticos.

29 Cf., p. e., (Heinrich, 1979). 
presentada a veces, de que la epistemología evolucionista constituye un reduccionismo científico o un círculo vicioso.

En todas las culturas han existido hipótesis explicativas del cosmos, de la vida, etc., que han tenido que ser producidas por innovaciones creativas. Cuando por primera vez en la historia, esas hipótesis fueron seguidas por la crítica, nació la "tradición crítica" —aparentemente un suceso único en la historia humana. Eso ocurrió con los presocráticos. ${ }^{30}$ Ellos fueron los Penates del racionalismo crítico.

En la epistemología popperiana la elección teórica se guía por buenas razones, basadas sobre reglas de evaluación extraídas de un ideal realista de la ciencia, es decir, de un ideal que no incluye la petición irrazonable (por ser imposible de satisfacer) de que los métodos de averiguar el valor de verdad de los enunciados descriptivos sean infalibles, es decir, nos proporcionen una garantía de verdad o nos garanticen una cierta probabilidad. Como hemos visto, el criticismo metodológico mostró que el ideal fundacionalista de la ciencia —el ideal del racionalismo clásico_era utópico, y por tanto, no podía proporcionar un principio regulador, lo que llevó a revisar la imagen del hombre subyacente en ese ideal.

Quisiera concluir con unas pocas conjeturas. La metodología falsacionista-evolucionista de Popper proporciona una herramienta utilísima al investigador, que le ayuda a mejorar su propia comprensión qua investigador y a racionalizar las decisiones a tomar, ofreciéndole ayuda en la conceptualización de los tipos de situaciones de investigación, por lo que crece en vez de disminuir su libertad de decisión, puesto que la decisión final se deja a su sensibilidad, y por consiguiente, le ofrece un instrumento muy manejable para estilizar la historia de la ciencia (res gestae) en una forma que tenga verdaderamente en cuenta la historicidad especial de la ciencia. Tiene en cuenta además el hecho de que, a través de la idea de progreso cognoscitivo - que es constitutiva del sentido de la ciencia- ésta se convierte en un fenómeno histórico y evolutivo en un sentido en el que el arte, la literatura, las instituciones... no lo son. Por medio de la idea de progreso cognoscitivo, la verdad o el parecido comparativo a la verdad queda reconocido como un principio regulador de la investigación científica. De esta forma, en esta epistemología caben tanto la historicidad esencial y evolutiva de la empresa científica como la verdad como un principio regulativo, aunque reconociendo siempre la falibilidad consustancial al intelecto humano. 


\section{BIBLIOGRAFIA}

Agassi, J. \& Cohen, R. S. (eds.). Scientific Philosophy Today. (Boston Studies in the Philosophy of Science). Dordrecht: Reidel, 1982.

Albert, H. Traktat über rationale Praxis. Tübingen: J. C. B. Mohr, 1978.

-. "Die Wissenschaft und die Suche nach Wahrheit" en (Radnitzky and Andersson, 1980), pp. 221-246.

Andersson, G. "Presuppositions, Problems, Progress", Introducción a (Radnitzky \& Andersson, 1979), pp. 3-13.

Andersson, G. "Sind Falsifikationismus und Fallibilismus vereinbar?", en (Radnitzky \& Andersson, 1981), pp. 255-276.

-. "Objective Criteria of Scientific Progress?", ver (Radnitzky \& Andersson, 1978).

Antiseri, D. Teoria unificata del metodo. Padova: Liviana, 1981.

Ayala, F. \& Dobzhansky, T. Studies in the Philosophy of Biology. New York: Macmillan, 1974.

Barone, F. "La contemporanea discussione metodologica e la storiografia delle scienza", Physis, 22: 191-209 (1980).

Bartley, W. W. The Retreat to Commitment. New York: Knopf, 1962.

-. Wittgenstein. Philadelphia and New York: Lippincott, 1973.

-. "The Philosophy of Karl Popper. Part I: Biology and Evolutionary Epistemology", Philosophia (Israel), 6: 463-494 (1976)..

Campbell, D. "Blind Variation and Selective Retention in Creative Thought as in Other Knowledge Processes", Psychological Review, 67: 380-400 (1960).

- "Evolutionary Epistemology" in (Schilpp, 1974), pp. 413-463 (1974a).

-. "Unjustified Variation and Selective Retention in Scientific Discovery" en (Ayala \& Dobzhansky, 1974), pp. 139-161 (1974b).

-. "Comment on 'The Natural Selection Model of Conceptual Evolution" ", Psychological Review, 44: 502-507 (1977).

Cohen, R., Feyerabend, P., and Wartofsky, M. (eds.). Essays in Memory of Imre Lakatos. (Boston Studies in the Philosophy of Science, vol. 39), Dordrecht: Reidel, 1976.

Feyerabend, P. "Consolations for the Specialist" en (Lakatos \& Musgrave, 1970), pp. 197-230.

-. "Von der beschränkten Gültigkeit methodologischer Regeln", Neue Hefte für Philosophie, vol. 1, 2-3: 124-171 (1972).

-. Against Method. Outline of an Anarchistic Theory of Knowledge. London: New Left Book, 1975.

- Probleme des Empirismus. Ausgewählte Schriften. Bd. 2, Braunschweig/ Wiesbaden: Vieweg, 1981.

Fich, H. Wittgenstein - the Later Philosophy. Atlantic Highlands, N. J.: Humanities, 1977.

-. "Wittgenstein and Popper", MS a publicarse en (International Cultural Foundation, 1982), en prensa.

Flew, A. Sociology, Equality and Education. London and New York: Macmillan, and Barnes and Noble, 1976.

-. A Rational Animal and Other Philosophical Essays on the Nature of Man. Oxford: Oxford University Press, 1978. 
-. "Commentary upon H. LeRoy Finch and G. Radnitzky", MS a publicarse en (International Cultural Foundation, 1982), en prensa.

Freeman, E. (ed.). The Abdication of Philosophy: Philosophy and the Public Good. Essays in Honor of Paul Arthur Schilpp. LaSalle, Ill.: Open Court, 1976.

Grmek, M., Cohen, R., \& Cimino, G. (eds.). On Scientific Dicovery. The Erice Lectures 1977. (Boston Studies in the Philosophy of Science, vol. 34), Dordrecht: Reidel, 1981.

Heirinch, B. Bumblebee Economics. Cambridge, Mass.: Harvard University Press, 1979.

Hempel, C. "Scientific Rationality: Normative vs. Descriptive Construals" en Wittgenstein, der Wiener Kreis und der kritische Rationalismus (Proceedings of the 3rd International Wittgenstein Symposium, August 1978), Wien, 1979, pp. 291-301.

International Cultural Foundation. The Search for Absolute Values and the Creation of the New World. (Proceedings of the Tenth International Conference on the Unity of the Sciences, Nov. 1981), New York, N. Y.: International Cultural Foundation Press, 1982 (en prensa).

Kuhn, T. S. The Structure of Scientific Revolutions. Chicago: Chicago University Press 1962, 2nd enl. ed. 1970.

-. "Logic of Discovery or Phychology of Research?" en (Lakatos \& Musgrave, 1970), pp. 1-23 (1970a).

- "Reflections on my Critics" en (Lakatos \& Musgrave, 1970), pp. 231-278 (1970b).

- The Essential Tension: Selected Studies in the Scientific Tradition and Change. Chicago: University of Chicago Press, 1977.

Lakatos, I. \& Musgrave, A. (eds.). Criticism and the Growth of Knowledge. London: Cambridge University Press, 1970.

Munz, P. The Shapes of Time. Middleton, Con.: Wesleyan University Press, 1977.

-. "Evolution and Equidistance", MS to be published (1981).

Popper, K. Logik der Forschung. Wien: Springer, 1934, 6 verb. Aufl. Tübingen: J. C. M. Mohr (Paul Siebeck), 1976.

-. The Open Society and its Enemies. 2 vols. London: Roudledge and Kegan Paul, 1945, 11 th. ed., 1977.

- Conjectures and Refutations. London: Routledge and Kegan Paul, 1963, 7 th rev. ed., 1978.

- The Logic of Scientific Discovery. London: Hutchison, 1959, 9th rev. ed., 1977 (trad. de Popper, 1934).

-. "The Myth of the Framework" in (Freeman, 1976), pp. 23-48.

-. Objective Knowledge. Oxford: The Clarendon Press, 1972, 5th rev. ed., 1979.

-. "Toleration and Intellectual Responsibility" (First J. B. Morrell Memorial Address, 13.3.81), MS inédito.

Radnitzky, G. "Popperian Philosophy of Science as an Antidote against Relativism" in (Cohen, Feyerabend, and Wartofsky, 1956), pp. 505-546.

-. "Justifying a Theory vs. Giving Good Reasons for Preferring a Theory. On the Big Divide in the Philosophy of Science" en (Radnitzky \& Anderson, 1979), pp. 213-256. 
-. "From Justifying a Theory to Comparing Theories and Selecting Questions", Revue Internationale de Philosophie, 34: 179-228 (1980).

-. "Progress and Rationality in Research" en (Grmek et al., 1981), pp. 43-102.

-. "Wertrreiheitsthese: Wissenschaft, Ethik und Politik" en (Radnitzky \& Anderson, 1981), pp. 47-126 (1981b).

-. "Analytic Philosophy as the Confrontation between Wittgensteinians and Popper" en (Agassi \& Cohen, 1981), pp. 239-286 (1981 c).

- "Dissappointment and Changes in the Conception of Rationality: Wittgenstein and Popper", MS a publicarse (International Cultural Foundation, 1982), en prensa (1982 a).

-. "Teoria della scienza e scienza come teoria" in Enciclopedia del Novecento, Vol. VI, Roma: Istituto della Enciclopedia Italiana, 1982, forthcoming (1982b).

Radnitzky, G. \& Andersson, G. "Objective Criteria of Scientific Progress? Inductivism, Falsificationism and Relativism" en (Radnitzky \& Andersson, 1978), pp. 3-19.

Radnitzky, G. \& Andersson, G. (eds.), Progress and Rationality in Science (Boston Studies in the Philosophy of Science, vol. 58), Dordrecht: Reidel, 1978.

-. The Structure and Development of Science (Boston Studies in the Philosophy of Science, vol. 59), Dordrecht: Reidel, 1979.

- Fortschritt und Rationalität der Wissenschaft. Tübingen: J. C. B. Mohr, 1980. (Rev. and enl. German version of (Randnitzky and Andersson, 1978)).

- Voraussetzungen und Grenzen der Wissenschaft. Tübingen: J. C. B. Mohr, 1981.

Rothbart, D. "Popper Against Inductivism", Dialectica, 34: 121-127 (1980).

Schilpp, P. (ed.). The Philosophy of Karl R. Popper. (The Library of Living Philosophers), 2 vols., LaSalle, Ill.: Open Court, 1974.

Wittgenstein, L. Tractatus Logico-Philosophicus. London: 1922, Routledge and Kegan Paul, 1961. 